International Journal of

Environmental Research and

Public Health

ISSN 1660-4601

www.mdpi.com/journal/ijerph

Article

\title{
Toxicity and Estrogenic Endocrine Disrupting Activity of Phthalates and Their Mixtures
}

\author{
Xueping Chen ${ }^{1}{ }^{*}$, Shisan $\mathrm{Xu}^{2}{ }^{2}$, Tianfeng Tan ${ }^{1}$, Sin Ting Lee ${ }^{1}$, Shuk Han Cheng ${ }^{2}$, \\ Fred Wang Fat Lee ${ }^{3}$, Steven Jing Liang $\mathrm{Xu}^{3}$ and Kin Chung $\mathrm{Ho}^{3}$
}

1 Vitargent (International) Biotechnology Limited, Unit 516, 5/F. Biotech Centre 2, No. 11 Science Park West Avenue, Hong Kong Science Park, Shatin, Hong Kong;

E-Mails: cindy.tan@vitargent.com (T.T.); Kathy.lee@ vitargent.com (S.T.L.)

2 State Key Laboratory in Marine Pollution, Department of Biology and Chemistry, City University of Hong Kong, 83 Tat Chee Avenue, Kowloon, Hong Kong; E-Mails: ssanyecao@ gmail.com (S.X.); bhcheng@cityu.edu.hk (S.H.C.)

3 School of Science and Technology, Open University of Hong Kong, 30 Good Shepherd Street, Homantin, Kowloon, Hong Kong; E-Mails: wflee@ouhk.edu.hk (F.W.F.L.);

sjlxu@ ouhk.edu.hk (S.J.L.X.); kcho@ouhk.edu.hk (K.C.H.)

* Author to whom correspondence should be addressed; E-Mail: xueping.chen@ hotmail.com; Tel.: +852-2393-2343; Fax: +852-2393-7922.

Received: 27 December 2013; in revised from: 28 February 2014 / Accepted: 4 March 2014 / Published: 14 March 2014

\begin{abstract}
Phthalates, widely used in flexible plastics and consumer products, have become ubiquitous contaminants worldwide. This study evaluated the acute toxicity and estrogenic endocrine disrupting activity of butyl benzyl phthalate (BBP), di(n-butyl) phthalate (DBP), bis(2-ethylhexyl) phthalate (DEHP), diisodecyl phthalate (DIDP), diisononyl phthalate (DINP), di- $n$-octyl phthalate (DNOP) and their mixtures. Using a $72 \mathrm{~h}$ zebrafish embryo toxicity test, the $\mathrm{LC}_{50}$ values of $\mathrm{BBP}, \mathrm{DBP}$ and a mixture of the six phthalates were found to be $0.72,0.63$ and $0.50 \mathrm{ppm}$, respectively. The other four phthalates did not cause more than $50 \%$ exposed embryo mortality even at their highest soluble concentrations. The typical toxicity symptoms caused by phthalates were death, tail curvature, necrosis, cardio edema and no touch response. Using an estrogen-responsive ChgH-EGFP transgenic medaka (Oryzias melastigma) eleutheroembryos based $24 \mathrm{~h}$ test, BBP demonstrated estrogenic activity, DBP, DEHP, DINP and the mixture of the six phthalates exhibited enhanced-estrogenic activity and DIDP and DNOP showed no enhanced- or anti-estrogenic
\end{abstract}


activity. These findings highlighted the developmental toxicity of BBP and DBP, and the estrogenic endocrine disrupting activity of BBP, DBP, DEHP and DINP on intact organisms, indicating that the widespread use of these phthalates may cause potential health risks to human beings.

Keywords: phthalate; toxicity; estrogenic endocrine disruptor; estrogenic activity; enhanced-estrogenic activity; mixture effects

\section{Introduction}

Phthalates are diesters of phthalic acids that are commonly used as plasticizer to increase the flexibility, pliability and elasticity of plastics, and also widely used in cosmetics, personal care products, food packaging and medical products [1] With annual production of about 6.0 million tons, phthalates have been detected in water [2], air [3], sediments [4], soil [5], food [6,7], human blood plasma [1], breast milk [8,9], urine [10] and so on. The large production volume and wide application has made the presence of phthalates almost ubiquitous. Phthalates were revealed to be obesogens in recent studies [11,12]. The phthalates dimethyl phthalate (DMP), diethyl phthalate (DEP), DBP, BBP, DEHP and DNOP are classified by the United States Environmental Protection Agency (USEPA) as priority environmental pollutants [13].

Mainly due to their endocrine disrupting potency, which causes adverse health effects, phthalates that used to be regarded as safe are now of wide concern. Some phthalates were reported to have the potential to cause decreased testicular weight and seminiferous tubular atrophy, increased DNA damage in men's sperm, premature breast development in girls, shortened pregnancy and decreased anogenital distance in newborn male babies (summarized by Schecter et al. [7]). These reproductive defects most likely result from the estrogen disrupting activity of certain phthalates.

Though studies have been carried out to evaluate the estrogenic endocrine disrupting activity of phthalates, and some phthalates such as DEP, DBP, BBP and DEHP have been reported to possess estrogenic activity in vitro [14-20], the toxicity and estrogenic endocrine disrupting potency of some phthalates and phthalate mixtures in in vivo systems are still not clear.

The present study used zebrafish (Danio rerio) embryos and estrogen-responsive ChgH-EGFP transgenic medaka (Oryzias melastigma) eleutheroembryos to evaluate the developmental toxicity and estrogenic endocrine disrupting potency of BBP, DBP, DEHP, DIDP, DINP, DNOP and their mixtures. These six phthalates are of concern for their potential migration from food containers into the food and are regulated for toys and childcare products by the European Union [21] and some other countries.

\section{Materials and Methods}

\subsection{Chemicals}

The phthalates used in this study are Butyl benzyl phthalate (BBP, CAS\# 85-68-7) from Aldrich, Di(n-butyl) phthalate (DBP, CAS\# 84-74-2), Di(2-ethylhexyl) phthalate (DEHP, CAS\# 117-81-7) and Diisodecyl phthalate (DIDP, CAS\# 26761-40-0) from CHEM Service, Diisononyl phthalate 
(DINP, CAS\# 28553-12-0) and Diisononyl phthalate (DNOP, CAS\# 117-84-0) from Wako. The chemical structures of these phthalates are shown in Figure 1. As phthalates are oily substances and cannot be properly dissolved in water, so methanol was used as vehicle to increase their solubility in water. The final concentration of vehicle in the exposure solutions was $0.1 \%$. 17- $\beta$-Estradiol (E2) standard was bought from the China National Standard Material Centre (Beijing, China). E2 stock solutions were prepared using methanol.

Figure 1. Chemical structure of phthalates.<smiles>CCCCOC(=O)c1ccccc1C(=O)OCc1ccccc1</smiles>

BBP

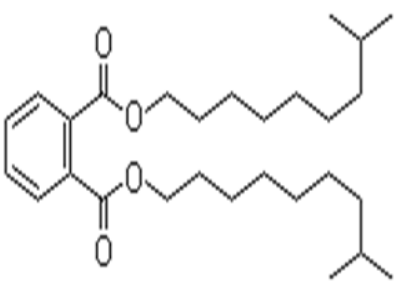

DIDP

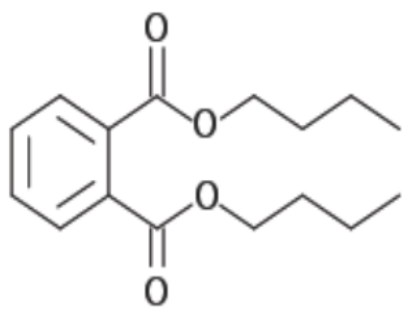

DBP

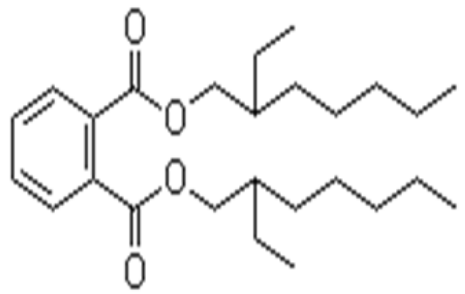

DINP

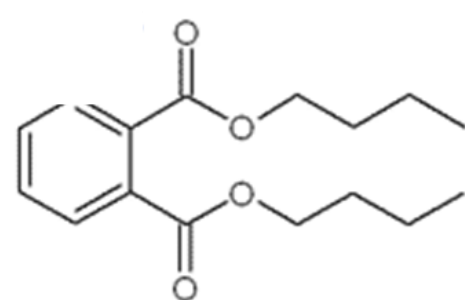

DEHP

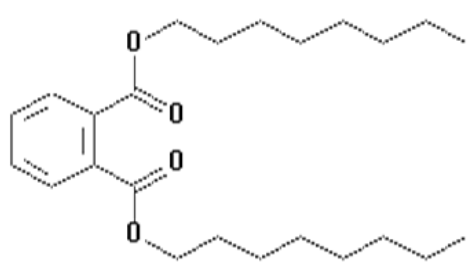

DNOP

\subsection{Zebrafish (Danio rerio) Maintenance and Zebrafish Embryo Toxicity Test}

Zebrafish AB strain was purchased from the Zebrafish International Resource Center (ZIRC) at the University of Oregon (Eugene, OR, USA). Adult zebrafish was maintained and fish embryos were collected as previously described [22]. Healthy embryos of 4-128 cell stages were used for the tests. Static exposure was carried out using 24-well plates. Each well contained one embryo, and each concentration was tested on 20 embryos. Phthalates and phthalate mixture (containing equal volumes of each phthalate at the stated concentration) were tested at 500.00, 100.00, 50.00, 10.00, 1.50, 0.60, 0.30, 0.06 and $0.01 \mathrm{ppm}$. Solvent $(0.1 \%$ methanol) and blank control were also tested. After $72 \mathrm{~h}$ exposure, embryos were observed under a stereomicroscope (Leica MZ10F, Leica, Hong Kong, China) and photographed using a CCD camera (Leica DFC 310 FX) connected to the stereomicroscope. The mortality rate of embryos exposed to each concentration was scored at the end of the test. The lethal concentration $50\left(\mathrm{LC}_{50}\right)$ was calculated based on the mortality-dose response curve. 


\subsection{Transgenic Medaka (Oryzias melastigma) Maintenance}

A stable transgenic $O$. melastigma strain containing the promoter of the estrogen-dependent liver-specific choriogenin $H(C h g H)$ gene regulating enhanced green fluorescence protein $(E G F P)$ gene coding region (ChgH-EGFP) was developed [23,24]. Transgenic medaka was cultured at $2.5 \mathrm{ppt}$ water at $26 \pm 1{ }^{\circ} \mathrm{C}$ with a constant $12 \mathrm{~h}$-light/12 h-dark photoperiod and fed with both brine shrimp (Artemia salina) and commercial flake food. Transgenic eleutheroembryos, the stage of hatched fish that still rely on the yolk for energy supply and have not started external feeding, were used for this study.

\subsection{Estrogenic Endocrine Disrupting Activity Test}

Based on the zebrafish embryo toxicity test results and solubility of phthalates in water, preliminary tests were performed to find out if individual phthalates and the phthalate mixture possessed estrogenic endocrine disrupting activity (including estrogenic activity, enhanced-estrogenic activity and anti-estrogenic activity). The concentrations used for preliminary test were 1.50, 0.60, 0.30, 0.06 and $0.01 \mathrm{ppm}$ for BBP and DBP, 50.00, 10.00, 1.50, 0.60, 0.30, 0.06 and $0.01 \mathrm{ppm}$ or DEHP, DIDP, DINP and DNOP, and 1.5, 0.06 and $0.01 \mathrm{ppm}$ for the phthalate mixture. For both estrogenic activity and enhanced-/anti-estrogenic activity pre-testing, about eight ChgH-EGFP transgenic medaka (O. melastigma) eleutheroembryos were exposed to each concentration of phthalate or phthalate mixture for $24 \mathrm{hrs}$ and these were then observed under a stereomicroscope (Leica MZ10F) equipped with a UV excitation light source and GFP filter set (excitation filter BP470/40 nm). If green fluorescence signal (GFP) was observed in the liver of exposed eleutheroembryos that means that the phthalate or phthalate mixture possesses estrogenic activity; if no GFP was observed in the liver of exposed eleutheroembryos that means that phthalate or phthalate mixture possess no estrogenic activity and their enhanced-/anti-estrogenic activity were then pre-tested. To test enhanced-/anti-estrogenic activity testing, E2 (e.g., 1.00 or $2.50 \mathrm{ppb}$ ) and E2 (e.g., 1.00 or $2.50 \mathrm{ppb}$ ) together with phthalate or phthalate mixture were tested and their induced GFP signal were observed and compared.

For easier comparison between different phthalates, one concentration (except for the phthalate mixture, which was tested at 0.15 and $1.50 \mathrm{ppm}$ ) that seems to induce, if there was any, an obvious signal was selected for formal testing. Formal testing exposures were performed using 24-well plates. Each concentration was tested in triplicate and each replicate contained eight eleutheroembryos. Methanol and water $(0.1 \%)$ were included as solvent and blank control, respectively. E2 solutions of $1.00,2.00,5.00$ and $10.00 \mathrm{ppb}$ were tested as positive reference. After $24 \mathrm{~h}$ exposure, the liver of eleutheroembryos were imaged using a CCD camera (Leica DFC 310 FX) connected to the fluorescence stereomicroscope (Leica MZ10F, Leia, Hong Kong, China). The grey value (brightness) of each eleutheroembryo's liver GFP signal intensity, represented as the relative fluorescence unit (RFU), were measured using image analysis software MetaMorph (Molecular Devices, Hong Kong, China). Data analysis were performed according to the ISO 20281 guidance [25] using Excel (Microsoft, Redmond, WA, USA). Student's $t$-test $(p<0.05)$ was performed to define the stastically significant enhanced-/anti-estrogenic activity of phthalate mixtures. 


\section{Results}

\subsection{Acute Toxicity of Phthalates and Their Mixtures}

The acute toxicity of phthalates and their mixtures to zebrafish embryos were analyzed in this study. The lethal concentration $50\left(\mathrm{LC}_{50}\right)$ of phthalates and their mixture were calculated basing on their mortality-dose response curve. Of the six phthalates, DBP is the most toxic, with $\mathrm{LC}_{50}$ of $0.63 \mathrm{ppm}$, followed by BBP, with $\mathrm{LC}_{50}$ of $0.72 \mathrm{ppm}$. Though DEHP, DIDP, DINP and DNOP caused toxicity symptoms at high concentrations, they did not resulted to more than half zebrafish embryos death even at their highest soluble concentrations (the highest soluble concentration is close to $500.00 \mathrm{ppm}$ for DEHP and $<500.00 \mathrm{ppm}$ for DIDP, DINP and DNOP) and their $\mathrm{LC}_{50}$ was not calculated. When the 6 phthalates were volume equally mixed together, additive toxicity effect was observed and its $\mathrm{LC}_{50}$ was calculated to be $0.50 \mathrm{ppm}$ (containing $0.50 \mathrm{ppm}$ of each of the six phthalates), more toxic than any of the 6 phthalates alone. The typical toxicity symptoms caused by phthalate included death, tail curvature, cardio edema, necrosis and no touch response (see Figure 2).

Figure 2. Representative photos of zebrafish (Danio rerio) embryos exposed to phthalates and phthalate mixture. (A): control; (B): 0.50 ppm BBP; (C): 1.50 ppm DBP; (D): mixture containing $0.50 \mathrm{ppm}$ of BBP, DBP, DEHP, DIDP, DIDP and DNOP, respectively. Representative toxicity symptoms caused by phthalates includes death (empty triangle), tail curvature (solid arrow), necrosis (empty arrow), cardio edema (arrowhead) and no touch response.
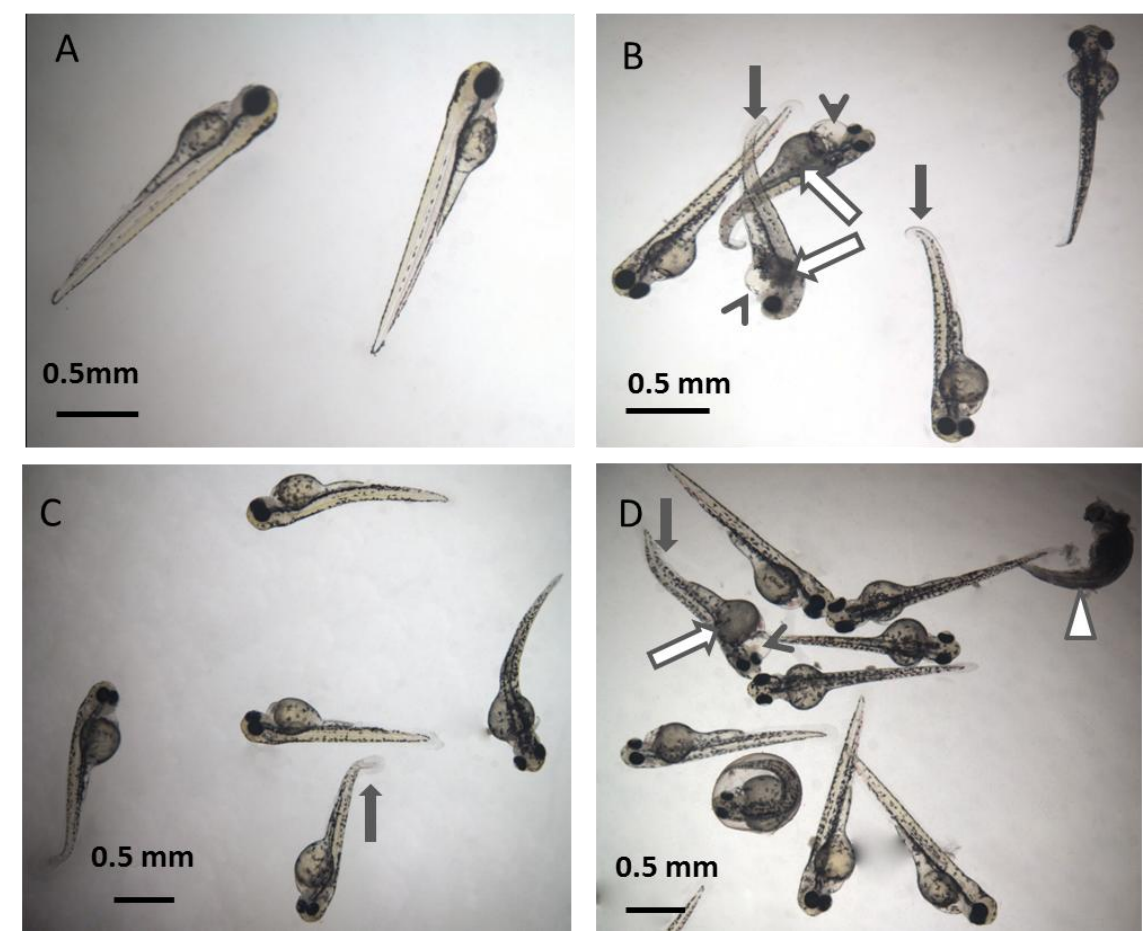

\subsection{Estrogenic Activity of Phthalates}

Basing on the zebrafish embryo acute toxicity test results the estrogenic activity of the 6 phthalates were screened and further tested using estrogen-responsive ChgH-EGFP transgenic medaka 
O. melastigma eleutheroembryos. Results showed that no GFP expression was induced in the liver of transgenic eleutheroembryos exposed to solvent and blank controls, and the GFP expression level, represented as relative fluorescence unit (RFU), induced by E2 was obviously dose-dependent (Figure 3). Of the 6 phthalates tested, only BBP (1.50 ppm) induced GFP expression and the intensity of which was close to that of $2.00 \mathrm{ppb}$ E2 (Figure 4). This means BBP possess estrogenic activity and is an estrogenic chemical.

Figure 3. Dose-dependent induction of GFP in transgenic medaka (O. melastigma) eleutheroembryos by $17 \beta$-estradiol (E2). Transgenic eleutheroembryos were exposed to varying concentrations of E2 for $24 \mathrm{hrs}$ and the induced GFP signal intensity (RFU) was measured (see Material and Methods for detail). No GFP was induced in solvent ( $0.1 \%$ methanol) and blank (0) controls.

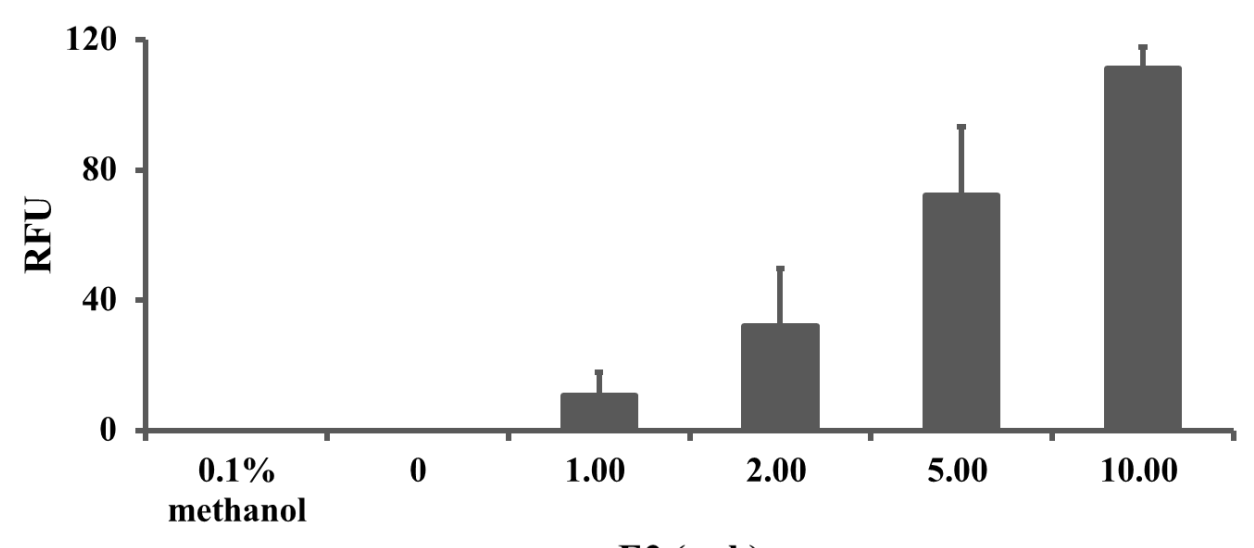

E2 (ppb)

Note: Data are mean \pm standard error of the mean.

Figure 4. Estrogenic activity of phthalate BBP. (A): BBP (1.50 ppm) induced GFP signal intensity (RFU) compared to that of E2 $(2.00 \mathrm{ppb})$, data are mean \pm standard error of the mean; (B): Representative photos of the livers of transgenic medaka (O. melastigma) eleutheroembryos after 24-h exposure to E2 (2.00 ppb) and BBP (1.50 ppm). Scale bar equals to $100 \mu \mathrm{m}$.

A

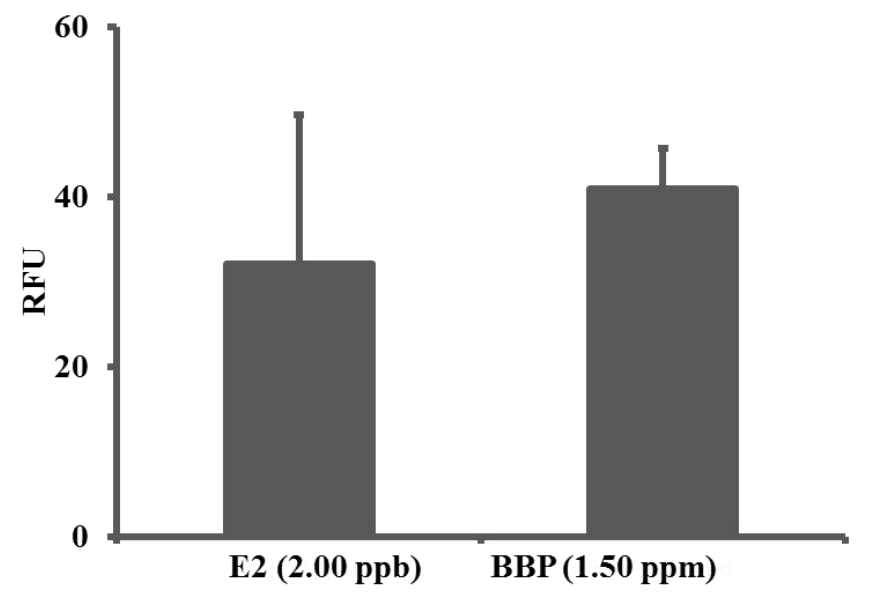

B

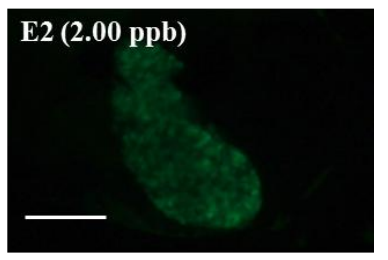

BBP (1.50 ppm) 


\subsection{Enhanced-/Anti-estrogenic Activity of Phthalates}

Phthalates DBP, DEHP, DIDP, DINP and DNOP that showed no estrogenic activity were processed for enhanced-/anti-estrogenic activity screening and testing. Transgenic eleutheroembryos were exposed to E2 (2.50 ppb) and E2 (2.50 ppb) together with phthalate for $24 \mathrm{~h}$, and their induced green fluorescence intensity (RFU) were measured and analyzed

Results showed that 1.00 ppm DBP, 1.50 ppm DEHP or DINP significantly increased the green fluorescence intensity (RFU) induced by E2, indicating the enhanced-estrogenic activity of the three phthalates (Figure 5). DIDP and DNOP did not significantly affect (increase or decrease) the GFP signal intensity (RFU) induced by E2 (Figure 5), meaning these two phthalates are not estrogenic endocrine disrupting compounds.

Figure 5. Enhanced-estrogenic activity of phthalates. DBP (1.00 ppm), DEHP (1.50 ppm) and DINP (1.50 ppm) significantly increased, and DIDP (1.5 ppm) and DNOP (1.5 ppm) did not statistically increase or decrease, the GFP signal intensity (RFU) of the livers of transgenic medaka (O. melastigma) eleutheroembryos induced by E2 (2.5 ppb).

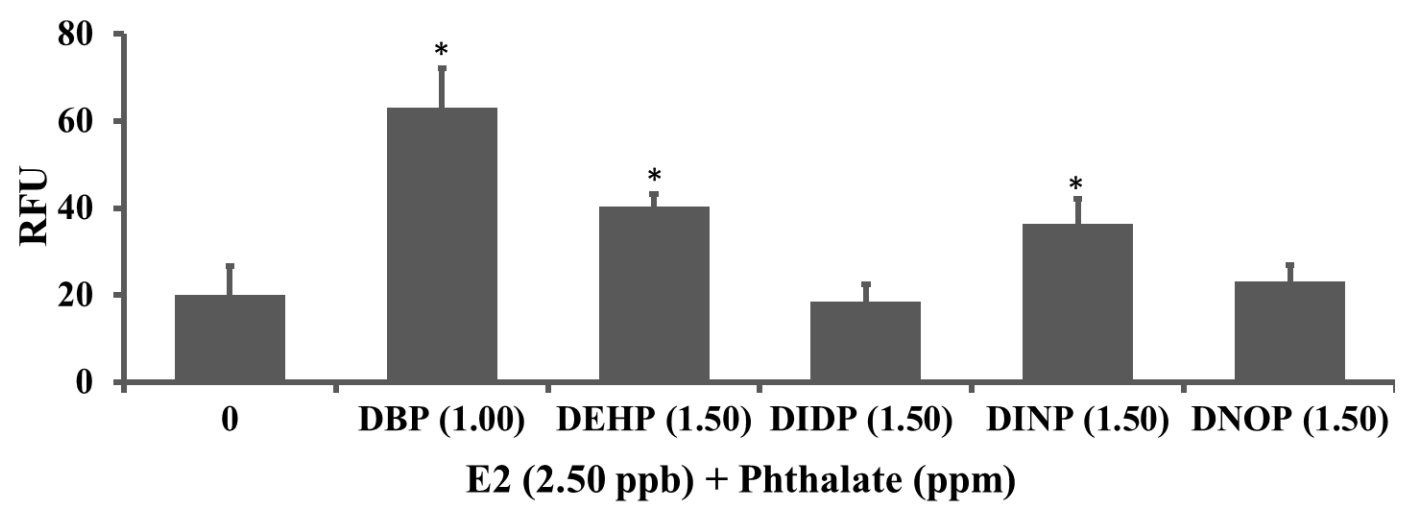

Notes: Data are mean \pm standard error of the mean; * denotes $p<0.05$.

\subsection{Estrogen Disrupting Activity of Phthalate Mixtures}

Further tests were performed to find out whether mixtures of the six phthalates have estrogenic or enhanced-/anti-estrogenic activity. Phthalates were mixed at the stated concentrations and tested. Figure 6A shows that phthalate mixtures at 0.15 and $1.50 \mathrm{ppm}$ induced very week GFP signals (RFU), demonstrating the weak estrogenic activity of the phthalate mixtures. However, when co-exposed with E2 (1 ppb), phthalate mixture at $1.50 \mathrm{ppm}$ mixture, but not $0.15 \mathrm{ppm}$, significantly increased the transgenic $O$. melastigma eleutheroembryos' liver GFP signal intensity (RFU). Representative photos of the livers of transgenic eleutheroembryos after $24 \mathrm{~h}$ exposure to E2 (1.00 ppb), phthalate mixture $(0.15 \mathrm{ppm})$ and $\mathrm{E} 2(1.00 \mathrm{ppb})+$ phthalate mixture $(1.50 \mathrm{ppm})$ respectively, are also presented (Figure 6B).

\section{Discussion and Conclusions}

Phthalates are ubiquitous in our daily life and may reach high concentrations in certain products and human populations. According to European Food Safety Authority regulation [26-30] regulation, 
the specific migration limits in food simulants are $30 \mathrm{mg} / \mathrm{kg}$ (i.e., $30 \mathrm{ppm}$ ), $0.3 \mathrm{mg} / \mathrm{kg}$ (i.e., $0.3 \mathrm{ppm}$ ) and $1.5 \mathrm{mg} / \mathrm{kg}$ (i.e., $1.5 \mathrm{ppm}$ ) for BBP, DBP and DEHP respectively and the compositional limits in food contact materials are $0.1 \%$ (i.e., 100 ppm) for BBP, DEHP, DIDP, DINP and DNOP and 0.05\% (i.e., $50 \mathrm{ppm}$ ) for DBP respectively. Based on these legislations, Danish scientists measured the contents of BBP, DBP, DEHP, DIDP and DINP in 100 officially collected food-contact material samples and found total 23 non-compliant samples.

Figure 6. Enhanced-estrogenic activity of phthalate mixtures. (A): The GFP signal intensity (RFU) induced by E2 (1.00 ppb), mixtures containing 0.15 and $1.50 \mathrm{ppm}$ of BBP, DBP, DEHP, DIDP, DINP and DNOP respectively, and E2 together with mixtures of 0.15 and $1.50 \mathrm{ppm}$ respectively. (B): Representative images of the livers of transgenic medaka (O. melastigma) eleutheroembryos after 24-h exposure to E2 (1.00 ppb), phthalate mixture $(0.15 \mathrm{ppm})$ and E2 (1.00 ppb) + phthalate mixture $(1.50 \mathrm{ppm})$ respectively.

$$
\text { A }
$$

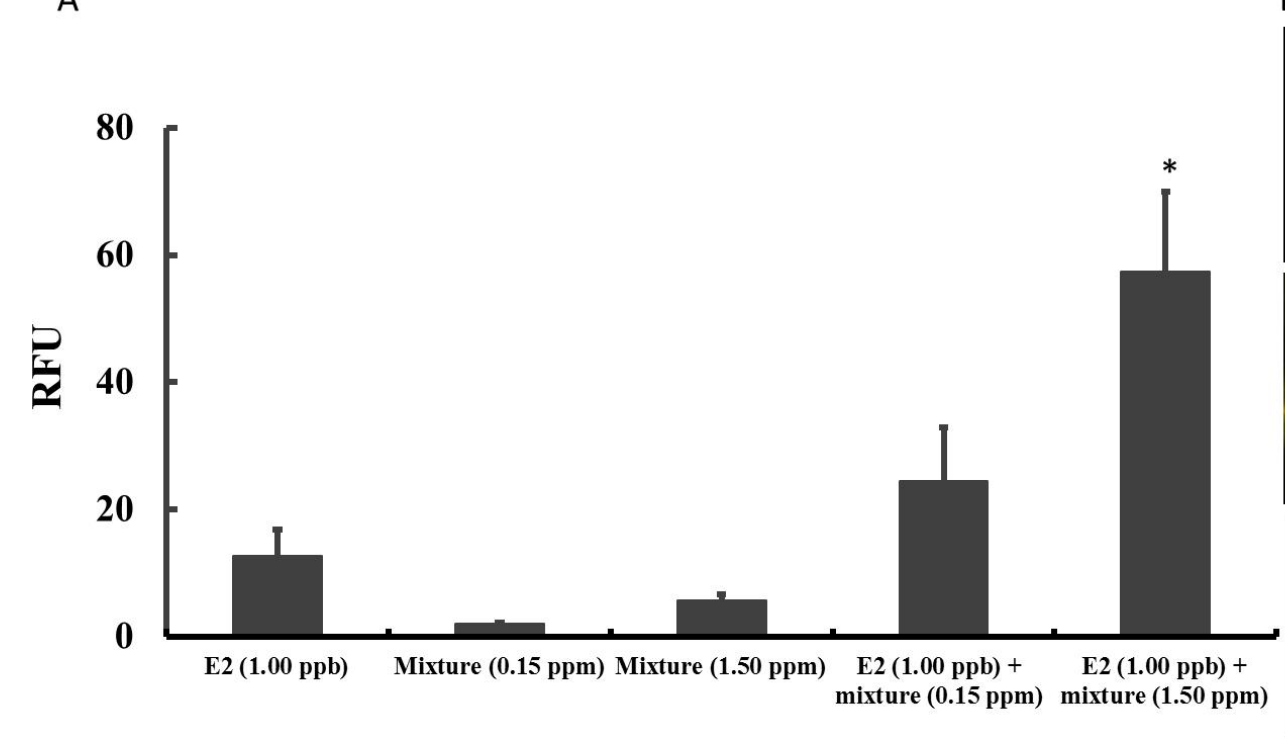

Notes: Data are mean \pm standard error of the mean; * denotes $p<0.05$; Scale bar equals to $100 \mu \mathrm{m}$.
B
$\mathrm{E} 2(1.00 \mathrm{ppb})$

Out of the 23 non-compliant samples, six samples contained $0.5 \%-5 \%$ DBP, 17 samples contained 0.05\%-50\% DEHP, five samples contained 25-50\% DIDP and five samples contained 5\%-50\% DINP [31]. In foods, BBP, DBP and DEHP were reported to be detected at a rate of 95.2\%, 90.5\%, and $90.5 \%$ in edible vegetable oil in USA [32] and DBP concentrations reached up to $20.76 \pm 6.60 \mathrm{ppb}$ and $66.78 \pm 7.60 \mathrm{ppb}$ in cow fresh milk and milk powder respectively [8]. In humans, DBP and DEHP were reported to be the most commonly detected phthalates in breast milk with concentrations ranging from low ppb level in Germany [9] up to dozens ppb level (57.78 $\pm 35.42 \mathrm{ppb}$ DBP) in China [8]; and in central Taiwan the total phthalate concentration in urine samples were reported to be $0.40 \mathrm{ppm}$ (geometric mean) for 2-years-olds and 5-years-olds and $0.20 \mathrm{ppm}$ (geometric mean) for pregnant women [10].

Using a 72-h zebrafish embryo acute toxicity test, this study demonstrated that BBP, DBP and a mixture of BBP, DBP, DEHP, DIDP, DINP and DNOP (i.e., containing $0.50 \mathrm{ppm}$ of each phthalate) possessed high toxicity, with $\mathrm{LC}_{50}$ values of $0.72 \mathrm{ppm}, 0.63 \mathrm{ppm}$ and $0.50 \mathrm{ppm}$, respectively, 
and induced severe developmental toxicity in live embryos. The high developmental toxicity of BBP and DBP revealed in present study and their reported comparatively high concentrations in the human body $[8,10]$ should raise concerns about their potential health risks to human beings, especially vulnerable groups such as pregnant women and children.

Besides acute toxicity, this study also evaluated the in vivo estrogenic and enhanced-estrogenic activity of the six phthalates and their mixtures using ChgH-EGFP transgenic Oryzias melastigma eleutheroembryos. Of the six tested phthalates, BBP exhibited detectable estrogenic activity, DBP, DEHP and DINP demonstrated enhanced-estrogenic activity, and DIDP and DNOP showed no enhanced- or anti-estrogenic activity. When the six phthalates were mixed at the same concentration, weak estrogenic activity and significant enhanced-estrogenic activity were detected at a $1.50 \mathrm{ppm}$ level. Comparing the data of Figures 5 and 6, no additive estrogenic activity or enhanced-estrogenic activity of DBP, DEHP and DINP was observed for mixtures at the tested concentrations. Of course, detailed phthalate mixture effects await further investigation.

The estrogenic endocrine disrupting activity test results of present study are generally consistent with that of previous reports, especially in vivo studies. To be more specific, BBP, though it caused no estrogenic effect in one ovariectomized Sprague-Dawley rat based an in vivo assay using uterine wet weight and vaginal cell cornification as endpoints [20], it has repeatedly demonstrated estrogenic activity in the present and several previous in vitro studies $[15,16,18,20]$. DBP that reportedly exhibited estrogenic activity in some in vitro tests [15,16,33], did not induce detectable estrogenic activity signal in the present and other in vitro and in vivo studies [20,34]. DEHP, like DBP, showed estrogenic activity in some in vitro and in vivo systems [19,35], but not in present and other in vitro and in vivo systems [20,33,36,37]. DIDP consistently showed no estrogenic activity in present and previous in vitro and in vivo assays [15,20]. DINP, except for very weak estrogenic activity exhibited in an in vitro assay [36] showed no estrogenic activity in the present and previous in vitro and in vivo tests [15,20]. DNOP, like DIDP, also consistently showed no estrogenic activity in present and previous in vitro and in vivo studies $[20,38]$. To conclude, phthalates that have been reported to possess estrogenic activity were identified to be either estrogenic (BBP), or enhanced-estrogenic (DBP, DEHP and DNOP) chemicals, and phthalates that consistently were reported to possess no estrogenic activity were further proved to be neither estrogenic nor enhanced-/anti-estrogenic chemicals in the present study.

The present study also reported the estrogenic and enhanced-estrogenic activity of phthalate mixtures. The results are in some sense consistent with that of a previous ER-luc transfected MVLN cell line study, which showed the mixture of BBP, DBP, dioctyl phthalate (DOP), DIDP, DINP, DEHP, bis(2-ethylhexyl) adipate (DEHA), 4-tert-octylphenol (tOP), 4-chloro-3-methylphenol (CMP), 2,4-dichlorophenol (2,5-DCP), 2-phenylphenol (2-PP) and resorcinol significantly induced the transactivation of ER in an additive manner [15]. Compared with a single chemical analysis, few studies have been carried out to investigate the enhanced-/anti-estrogenic activity of mixtures, which may be explained by several reasons including, but not limited to, the unavailability of proper testing methods, the complexity of chemical interactions in mixtures, and the difficulty of mimicking real situations.

In conclusion, using two fish models, this study demonstrated the comparatively high toxicity of BBP and DBP, confirmed the estrogenic activity of BBP, and revealed the enhanced-estrogenic 
activity of DBP, DEHP, DINP and mixture of BBP, DBP, DEHP, DIDO, DINO and DNOP. This study also demonstrated the potential of using genetically modified fish eleutheroembryos for efficient estrogenic endocrine disruptors testing.

\section{Acknowledgments}

This study was partially supported by the State Key Laboratory in Marine Pollution of City University of Hong Kong.

\section{Author Contributions}

The work present here was carried out in collaboration between all authors. Xueping Chen, Shuk Han Cheng and Kin Chung Ho confined the research theme and Xueping Chen wrote the paper. Shisan $\mathrm{Xu}$ designed methods and experiments, carried out the experiments, analyzed the data. Tianfeng Tan, Sin Ting Lee, Fred Wang Fat Lee and Steven Jing Liang Xu co-designed experiments and co-worked on data analyses, interpretation and presentation. All authors have contributed to, read and approved the manuscript.

\section{Conflicts of Interest}

The authors declare no conflict of interest.

\section{References}

1. Wan, H.T.; Leung, P.Y.; Zhao, Y.G.; Wei, X.; Wong, M.H.; Wong, C.K. Blood plasma concentrations of endocrine disrupting chemicals in Hong Kong populations. J. Hazard. Mater. 2013, 261, 763-769.

2. Xie, Z.; Ebinghaus, R.; Temme, C.; Lohmann, R.; Caba, A.; Ruck, W. Occurrence and air-sea exchange of phthalates in the Arctic. Environ. Sci. Tech. 2007, 41, 4555-4560.

3. Wang, W.X.; Zhang, Y.L.; Wang, S.L.; Fan, C.Q.; Xu, H. Distributions of phthalic esters carried by total suspended particulates in Nanjing, China. Environ. Monit. Assess. 2012, 184, 6789-6798.

4. Liu, H.; Liang, H.; Liang, Y.; Zhang, D.; Wang, C.; Cai, H.; Shvartsev, S.L. Distribution of phthalate esters in alluvial sediment: A case study at JiangHan Plain, Central China. Chemosphere 2010, 78, 382-388.

5. Kong, S.F.; Ji, Y.Q.; Liu, L.L.; Chen, L.; Zhao, X.Y.; Wang, J.J.; Bai, Z.P.; Sun, Z.R. Diversities of phthalate esters in suburban agricultural soils and wasteland soil appeared with urbanization in China. Environ. Pollut. 2012, 170, 161-168.

6. Fierens, T.; Van Holderbeke, M.; Willems, H.; De Henauw, S.; Sioen, I. Transfer of eight phthalates through the milk chain-A case study. Environ. Int. 2013, 51, 1-7.

7. Schecter, A.; Lorber, M.; Guo, Y.; Wu, Q.; Yun, S.H.; Kannan, K.; Hommel, M.; Imran, N.; Hynan, L.S.; Cheng, D.; Colacino, J.A.; Birnbaum, L.S. Phthalate concentrations and dietary exposure from food purchased in New York State. Environ. Health Perspect. 2013, 121, 473-494.

8. Liu, H.J.; Cao, J.; Shu, W.Q. Comparison of organic component and di-n-butyl phthalate between human milk and cow milk products (in Chinese). Zhonghua Yu Fang Yi Xue Za Zhi. 2011, 45, 53-56. 
9. Zimmermann, S.; Gruber, L.; Schlummer, M.; Smolic, S.; Fromme, H. Determination of phthalic acid diesters in human milk at low ppb levels. Food Addit. Contam. Pt. A 2012, 29, 1780-1790.

10. Lin, S.; Ku, H.Y.; Su, P.H.; Chen, J.W.; Huang, P.C.; Angerer, J.; Wang, S.L. Phthalate exposure in pregnant women and their children in central Taiwan. Chemosphere 2011, 82, 947-955.

11. Hao, C.; Cheng, X.; Guo, J.; Xia, H.; Ma, X. Perinatal exposure to diethyl-hexyl-phthalate induces obesity in mice. Front. Biosci. 2013, 5, 725-733.

12. Pereira-Fernandes, A.; Demaegdt, H.; Vandermeiren, K.; Hectors, T.L.; Jorens, G.; Blust, R.; Vanparys, C. Evaluation of a screening system for obesogenic compounds: Screening of endocrine disrupting compounds and evaluation of the PPAR dependency of the effect. PLoS One 2013, 8, doi:10.1371/journal.pone.0077481.

13. United States Environmental Protection Agency (USEPA). Electronic Code of Federal Regulations. Available online: http://www.ecfr.gov/cgi-bin/text-idx?c1/4ecfr\&SID1/4b960051a53 c9015d817718d71f1617b7\&rgn1/4div5\&view1/4text\&node1/440,30.0.1.1.23\&idno $1 / 440 \# 40: 30.0 .1 .1$. 23.0.5.9.9. (accessed on 13 March 2014).

14. Andersen, H.R.; Andersson, A.M.; Arnold, S.F.; Autrup, H.; Barfoed, M.; Beresford, N.A.; Bjerregaard, P.; Christiansen, L.B.; Gissel, B.; Hummel, R.; et al. Comparison of short-term estrogenicity tests for identification of hormone-disrupting chemicals. Environ. Health Perspect. 1999, 107, 89-108.

15. Ghisari, M.; Bonefeld-Jorgensen, E.C. Effects of plasticizers and their mixtures on estrogen receptor and thyroid hormone functions. Toxicol. Lett. 2009, 189, 67-77.

16. Ho, P.W.; Tse, Z.H.; Liu, H.F.; Lu, S.; Ho, J.W.; Kung, M.H.; Ramsden, D.B.; Ho, S.L. Assessment of cellular estrogenic activity based on estrogen receptor-mediated reduction of soluble-form catechol-o-methyltransferase (COMT) expression in an ELISA-based system. PLoS One 2013, 8, doi:10.1371/journal.pone.0074065.

17. Jobling, S.; Reynolds, T.; White, R.; Parker, M.G.; Sumpter, J.P. A variety of environmentally persistent chemicals, including some phthalate plasticizers, are weakly estrogenic. Environ. Health Perspect. 1995, 103, 582-587.

18. Parveen, M.; Inoue, A.; Ise, R.; Tanji, M.; Kiyama, R. Evaluation of estrogenic activity of phthalate esters by gene expression profiling using a focused microarray (estrarray ${ }^{\circledR}$ ). Environ. Toxicol. Chem. 2008, 27, 1416-1425.

19. Veld, T.M.G.; Zawadzka, E.; van den Berg, J.H.; van der Saag, P.T.; Rietjens, I.M.; Murk, A.J. Food-associated estrogenic compounds induce estrogen receptor-mediated luciferase gene expression in transgenic male mice. Chem. Biol. Interact. 2008, 174, 126-133.

20. Zacharewski, T.R.; Meek, M.D.; Clemons, J.H.; Wu, Z.F.; Fielden, M.R.; Matthews, J.B. Examination of the in vitro and in vivo estrogenic activities of eight commercial phthalate esters. Toxicol. Sci. 1998, 46, 282-293.

21. Directive 2005/84/EC of the European Parliament and of the Council of 14 December 2005 Amending for the 22nd Time Council Directive 76/769/EEC on the Approximation of the Laws, Regulations and Administrative Provisions of the Member States Relating to Restrictions on the Marketing and Use of Certain Dangerous Substances and Preparations (Phthalates in Toys and Childcare Articles). Available online: http://eur-lex.europa.eu/LexUriServ/LexUriServ.do?uri=OJ:L: 2005:344:0040:0043:en:PDF (accessed on 13 March 2014). 
22. Cheng, S.H.; Wai, A.W.K.; So, C.H.; Wu, R.S.S. Cellular and molecular basis of cadmium-induced deformities in zebrafish embryos. Environ. Toxic. Chem. 2000, 19, 3024-3031.

23. Chen, X.; Kinoshita, M.; Hirata, T.; Yu, R.M.K.; Cheng, S.H. Transgenic marine medaka (Oryzias melastigma): A sensitive sentinel for estrogenic pollutants. Mol. Cell Toxicol. 2007, 3. Available online: http://astp.jst.go.jp/modules/search/DocumentDetail/1738-642x\%2B\%2540\% 2B_3_4_Transgenic\%2BMarine\%2BMedaka\%2B\%2528Oryzias\%2BMelastigma\%2529\%253A $\%$ 2Ba\%2BSensitive\%2BSentinel\%2Bfor\%2BEstrogenic\%2BPollutants_N\%252FA (accessed on 13 March 2014).

24. Cheng, S.H.; Chen, X. Transgenic Fish and Uses Thereof. U.S. Patent Number US8395018 B2, 12 March 2013.

25. ISO/TS 20281:2006. Water quality - Guidance on statistical interpretation of ecotoxicity data.

26. European Food Safety Authority (EFSA). Opinion of the scientific panel on food additives, flavourings, processing aids and materials in contact with food (AFC) on a request from the commission related to butylbenzylphthalate (BBP) for use in food contact materials. EFSA J. 2005, 241, 1-14.

27. European Food Safety Authority (EFSA). Opinion of the scientific panel on food additives, flavourings, processing aids and materials in contact with food (AFC) on a request from the commission related to bis(2-ethylhexyl) phthalate (DEHP) for use in food contact materials. EFSA J. 2005, 243, 1-20.

28. European Food Safety Authority (EFSA). Opinion of the scientific panel on food additives, flavourings, processing aids and materials in contact with food (AFC) on a request from the commission related to di-isodecylphthalate (DIDP) for use in food contact materials. EFSA J. 2005, 245, 1-14.

29. European Food Safety Authority (EFSA). Opinion of the scientific panel on food additives, flavourings, processing aids and materials in contact with food (AFC) on a request from the commission related to di-isononylphthalate (DINP) for use in food contact materials. EFSA J. 2005, 244, 1-18.

30. European Food Safety Authority (EFSA). Opinion of the scientific panel on food additives, flavourings, processing aids and materials in contact with food (AFC) on a request from the Commission related to di-butylphthalate (DBP) for use in food contact materials. EFSA J. 2005, 242, 1-17.

31. Petersen, J.H.; Jensen, L.K. Phthalates and food-contact materials: enforcing the 2008 European Union plastics legislation. Food Addit. Contam. Part A Chem. Anal. Control Expo. Risk Assess. 2010, 27, 1608-1616.

32. Bi, X.; Pan, X.; Yuan, S.; Wang, Q. Plasticizer contamination in edible vegetable oil in a U.S. retail market. J. Agric. Food Chem. 2013, 61, 9502-9509.

33. Shen, O.; Du, G.; Sun, H.; Wu, W.; Jiang, Y.; Song, L.; Wang, X. Comparison of in vitro hormone activities of selected phthalates using reporter gene assays. Toxicol. Lett. 2009, 191, 9-14.

34. Filipiak, E.; Walczak-Jędrzejowska, R.; Krupiński, M.; Oszukowska, E.; Marchlewska, K.; Długoński, J.; Kula, K.; Słowikowska-Hilczer, J. Di(n-butyl) phthalate has no effect on the rat prepubertal testis despite its estrogenic activity in vitro. Folia Histochem. Cytobiol. 2011, 49, 685-689. 
35. Nomura, Y.; Mitsui, N.; Bhawal, U.K.; Sawajiri, M.; Tooi, O.; Takahashi, T.; Okazaki, M. Estrogenic activity of phthalate esters by in vitro VTG assay using primary-cultured Xenopus hepatocytes. Dent. Mater. J. 2006, 25, 533-537.

36. Harris, C.A.; Henttu, P.; Parker, M.G.; Sumpter, J.P. The estrogenic activity of phthalate esters in vitro. Environ. Health Perspect. 1997, 105, 808-811.

37. Zhang, Y.; Chen, B.; Ding, X.; Xie, X. Potential estrogenic activities of DBP, DEHP and their metabolites in immature rats using the uterotrophic assay. J. Environ. Occup. Med. 2005, 22, 11-13.

38. ECHA (European Chemical Agency). Evaluation of New Scientific Evidence Concerning the Restrictions Contained in Annex XVII to Regulation (EC) No. 1907/2006 (REACH). Available online: http://echa.europa.eu/documents/10162/a35fa99b-ed8f-4451-a4d5-f012e9ba69c7 (accessed on 13 March 2014).

(C) 2014 by the authors; licensee MDPI, Basel, Switzerland. This article is an open access article distributed under the terms and conditions of the Creative Commons Attribution license (http://creativecommons.org/licenses/by/3.0/). 